\title{
Opportunities to Foster Efficient Communication in Labor and Delivery Using Simulation
}

\author{
Kay Daniels, MD ${ }^{1,2}$ Colleen Hamilton ${ }^{2}$ Susan Crowe, $\mathrm{MD}^{1,2}$ Steven S. Lipman, MD ${ }^{2,3}$ \\ Louis P. Halamek, MD ${ }^{2,4}$ Henry C. Lee, MD $^{2,4}$
}

${ }^{1}$ Department of Obstetrics and Gynecology, Stanford University School of Medicine, Stanford, California

${ }^{2}$ Department of Pediatrics, Safety Learning Laboratory for Neonatal and Maternal Care, Stanford University, Stanford, California

${ }^{3}$ Department of Anesthesiology, Pain and Perioperative Medicine,

Stanford University School of Medicine, Stanford, California

${ }^{4}$ Department of Pediatrics, Stanford University School of Medicine, Stanford, California

Am J Perinatol Rep 2017;7:e44-e48.

\begin{abstract}
Address for correspondence Henry Lee, MD, Division of Neonatal and Developmental Medicine, Department of Pediatrics, Stanford University School of Medicine, 1265 Welch Road, Stanford, CA 94305 (e-mail: hclee@stanford.edu).
\end{abstract}

\begin{abstract}
Keywords

- communication

- health care professionals

- labor and delivery

- simulation

Introduction Communication errors are an important contributing factor in adverse outcomes in labor and delivery (L\&D) units. The objective of this study was to identify common lapses in verbal communication using simulated obstetrical scenarios and propose alternative formats for communication.

Methods Health care professionals in L\&D participated in three simulated clinical scenarios. Scenarios were recorded and reviewed to identify questions repeated within and across scenarios. Questions that were repeated more than once due to ineffective communication were identified. The frequency with which the questions were asked across simulations was identified.

Results Questions were commonly repeated both within and across 27 simulated scenarios. The median number of questions asked was 27 per simulated scenario. Commonly repeated questions focused on three general topics: (1) historical data/ information (i.e., estimated gestational age), (2) maternal clinical status (i.e., estimated blood loss), and (3) personnel (i.e., "Has anesthesiologist been called?").

Conclusion Inefficient verbal communication exists in the process of transferring information during obstetric emergencies. These findings can inform improved training and development of information displays to improve teamwork and communication. A visual display that can report static historical information and specific dynamic clinical data may facilitate optimal human performance.
\end{abstract}

Labor and delivery (L\&D) units are acute care units where life's most joyful moments transpire, but unfortunately, they are not immune to adverse and sometimes tragic outcomes. A 2004 Sentinel Event Alert published online by the Joint Commission indicated that communication errors in the delivery room were the primary root cause of $72 \%$ of the neonatal deaths and long-term neurologic morbidity reported to that agency. ${ }^{1,2}$

Extensive research and development in the area of medical team communication has led to the development of received

August 4, 2016

accepted after revision

January 9, 2017
DOI http://dx.doi.org/

$10.1055 / \mathrm{s}-0037-1599123$.

ISSN 2157-6998.
Copyright $\odot 2017$ by Thieme Medical Publishers, Inc., 333 Seventh Avenue, New York, NY 10001, USA.

Tel: +1(212) 584-4662.
License terms

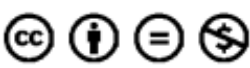


TeamSTEPPS and similar training programs based on strategies originally developed for commercial aviation and other high-risk industries (e.g., Crew Resource Management). ${ }^{3-7}$ These tools have provided a roadmap for improving team communication with the use of check backs and closed loop techniques.

However, despite this progress in strategies to improve health care team performance, the etiology of gaps in teamwork and communication in acute situations similar to those that occur in L\&D units are not well known. The objective of this study was to use simulated scenarios to identify common lapses in communication during high-risk scenarios in the L\&D setting by exploring the types and frequency of questions asked during such scenarios.

\section{Methods}

The setting of this study was a L\&D unit at a tertiary care obstetric center (Lucile Packard Children's Hospital Stanford, Stanford, CA). As part of ongoing continuing education and quality improvement, a multidisciplinary team of health care professionals (HCPs) participated in routine simulation exercises in situ (in the actual L\&D setting where patient care occurs), using a sophisticated human patient simulator (Noelle, Gaumard) in scenarios conducted by HCPs skilled in the use of simulation.

The teams of subjects consisted of four to five L\&D nurses, one obstetric technician, one to two anesthesiologists, and two obstetricians. All participants were at a minimal level postgraduate year 3 or greater for residents and all the L\&D nurses and technicians had experience on L\&D. No students were involved. Simulated clinical scenarios have been embedded in team training in this L\&D unit since 2004; thus, the majority of participants have experienced a minimum of 2 to 3 years of biannual simulation-based learning opportunities.

A series of simulated clinical scenarios involving three common obstetric emergencies were utilized in this study: (1) postpartum hemorrhage ( $\mathrm{PPH})$; (2) maternal code/cardiorespiratory arrest; and (3) severe preeclampsia. Each scenario lasted approximately 15 to 20 minutes. Participants were instructed to perform all the usual actions including calling for additional staff. The simulation team responded to all requests and provided the equipment or services in the usual time frame as in a real event. The goal of video review was to evaluate the questions asked during scenarios, specifically, (1) which questions were asked across multiple scenarios and (2) which questions were repeatedly asked within the same scenario. One investigator reviewed all of the videos, with initial guidance and review of scenarios from two of the other investigators. Only questions in which the HCP asking the question did not know the answer to the question were considered. Thus, rhetorical questions were not counted for the analysis.

The obstetric simulations evaluated for this study were a routine aspect of quality assurance and quality improvement at our institution and no human subjects could be harmed. The research protocol was considered as exempt by the Stanford University Institutional Review Board.

\section{Results}

A total of 27 simulations were conducted and reviewed, with 9 each of PPH, maternal cardiorespiratory arrest, and severe preeclampsia. There were similar numbers of total questions and questions repeated across the three scenario types, with maternal arrest eliciting the most questions (-Table 1). The median number of questions asked during a simulation scenario was 27 across all scenarios.

The types of questions asked during scenarios were often specific to the clinical circumstance. However, there were some questions that were common across scenarios (-Table 2). For example, "Has someone called anesthesiologist?" was asked in $78 \%$ of PPH scenarios and $100 \%$ of preeclampsia scenarios. The question was also commonly repeated within the same scenario in both of those clinical conditions. Similarly, the question of whether a pediatrician or neonatologist was called was commonly asked in both the maternal arrest and preeclampsia scenarios.

Aside from questions regarding personnel, clinical questions were asked about the mother's history (gestational age, history of high blood pressure) as well as dynamic questions about ongoing care in terms of therapies provided and current status of the mother.

Some questions were asked in a repeated fashion within the same scenario (-Table 3 ). In particular, questions regarding the status of personnel tended to be repeated. For example, "Has someone called anesthesiologist?" was asked multiple times within the same scenario in $43 \%$ of $\mathrm{PPH}$

Table 1 Prevalence of repeated questions within obstetric simulations

\begin{tabular}{|l|l|l|l|l|}
\hline Clinical scenario & $N$ (simulations) & $\begin{array}{l}\text { Median number of } \\
\text { questions asked } \\
\text { (interquartile range) }\end{array}$ & $\begin{array}{l}\text { Median number of } \\
\text { repeated questions } \\
\text { (interquartile range) }\end{array}$ & $\begin{array}{l}\text { Percentage of } \\
\text { questions that were } \\
\text { repeated per scenario } \\
\text { (interquartile range) }\end{array}$ \\
\hline $\begin{array}{l}\text { Postpartum } \\
\text { hemorrhage }\end{array}$ & 9 & $22(19-23)$ & $7(4-9)$ & $28 \%(24-42 \%)$ \\
\hline Maternal code & 9 & $35(28-37)$ & $9(4-14)$ & $29 \%(14-35 \%)$ \\
\hline Preeclampsia & 9 & $27(20-31)$ & $8(4-11)$ & $33 \%(27-35 \%)$ \\
\hline All combined & 27 & $27(20-35)$ & $8(4-11)$ & $30 \%(19-39 \%)$ \\
\hline
\end{tabular}


Table 2 Most common questions asked during multiple obstetric simulations

\begin{tabular}{|c|c|c|c|}
\hline Question & $\begin{array}{l}\mathrm{PPH} \\
N=9\end{array}$ & $\begin{array}{l}\text { Maternal code } \\
N=9\end{array}$ & $\begin{array}{l}\text { Preeclampsia } \\
N=9\end{array}$ \\
\hline \multicolumn{4}{|l|}{ Personnel } \\
\hline Has someone called anesthesiologist? & $7(78 \%)$ & & $9(100 \%)$ \\
\hline Has someone called pediatrician/NICU nurse? & & $9(100 \%)$ & $5(56 \%)$ \\
\hline Have we called/can you call a code (for help)? & & $8(89 \%)$ & \\
\hline Is someone timing/keeping record? & & $7(78 \%)$ & \\
\hline \multicolumn{4}{|l|}{ Equipment } \\
\hline Do you have an epidural? & $6(67 \%)$ & & \\
\hline Have we called for a PPH kit? & $5(56 \%)$ & & \\
\hline Can we start another IV? & $4(44 \%)$ & & \\
\hline Can we have an intrauterine tamponade device? & $4(44 \%)$ & & \\
\hline Has a Foley been placed/Is the Foley in? & & $7(78 \%)$ & \\
\hline [Where is the] crash cart? & & $6(67 \%)$ & \\
\hline \multicolumn{4}{|l|}{ Clinical information-dynamic } \\
\hline Is the placenta still in/has the placenta delivered? & $6(67 \%)$ & & \\
\hline What is the estimated blood loss? & $5(56 \%)$ & & \\
\hline How long ago was the child born/what was the delivery time? & $5(56 \%)$ & & \\
\hline Bleeding/hypertension during the delivery? & $3(33 \%)$ & & $4(44 \%)$ \\
\hline Are there contractions? & & & $6(67 \%)$ \\
\hline Is there fever? & & & $2(22 \%)$ \\
\hline \multicolumn{4}{|l|}{ Clinical information-static } \\
\hline History of high blood pressure? & & & $9(100 \%)$ \\
\hline Problems with vision? & & & $7(78 \%)$ \\
\hline Gestational age? & & & $7(78 \%)$ \\
\hline Is this their first infant? & & & $4(44 \%)$ \\
\hline When did the stomach pain start? & & & $2(22 \%)$ \\
\hline
\end{tabular}

Abbreviations: IV, intravenous; NICU, neonatal intensive care unit; PPH, postpartum hemorrhage.

scenarios, and "Has someone called pediatrician/neonatal intensive care unit nurse?" was repeatedly asked in 56\% of maternal code and $40 \%$ of preeclampsia scenarios (-Table 3 ).

\section{Discussion}

This study used videotaped simulated clinical scenarios to analyze the number and types of requests for information among HCPs during obstetrical emergencies. Although it is inevitable that questions would be asked during these emergencies, it is notable that questions are often repeated in any individual clinical scenario. Furthermore, we found that there were with identical requests for information being repeated across all three simulated L\&D emergency scenarios.

Three general categories of questions were noted across all of the clinical conditions studied. The first category involved static medical information such as gestational age and maternal history. The second category focused on dynamic medical information, such as maternal clinical status, including but not limited to vital signs and estimated blood loss. The third category of questions was focused on staffing/ personnel resources. Emergencies on L\&D often involve multiple teams from different service lines (obstetrics, anesthesia, and neonatology/pediatrics). Individual team members may be responding from different areas of the hospital; therefore, the availability and presence of personnel was a frequent question across clinical conditions, and sometimes repeated within one simulated clinical scenario.

The strengths of our study include performance of all the simulations on the actual L\&D unit in which the teams rendered care (in situ simulations) rather than in a simulation laboratory. Our teams were all experienced HCPs and intimately familiar with the location of equipment, supplies, configurations of the rooms, and the additional resources available. As a result, simulation artifact introduced by practicing scenarios in foreign environments or with inexperienced HCP was not relevant in these scenarios. Further, we used the same multidisciplinary teams that would be responding together to these emergencies if an actual event 
Table 3 Questions repeated during obstetric simulations within the same episode

\begin{tabular}{|l|l|l|l|}
\hline Question & PPH & Maternal code & Preeclampsia \\
\hline Personnel & $3 / 7(43 \%)$ & & $4 / 9(44 \%)$ \\
\hline Has someone called anesthesiologist? & & $5 / 9(56 \%)$ & $2 / 5(40 \%)$ \\
\hline Has someone called pediatrician/NICU nurse? & & $5 / 8(63 \%)$ & \\
\hline Have we called/can you call a code (for help)? & & $1 / 7(14 \%)$ & \\
\hline Is someone timing/keeping record? & & \\
\hline Equipment & & \\
\hline Do you have an epidural? & $1 / 6(17 \%)$ & \\
\hline Have we called for a PPH kit? & $3 / 5(60 \%)$ & \\
\hline Can we start another IV? & $3 / 4(75 \%)$ & & \\
\hline Can we have an intrauterine tamponade device? & $1 / 4(25 \%)$ & \\
\hline Has a Foley been placed/is the Foley in? & & $3 / 7(43 \%)$ \\
\hline [Where is the] crash cart? & $3 / 5(60 \%)$ & \\
\hline Clinical information-dynamic & \\
\hline Is the placenta still in/has the placenta delivered? & & \\
\hline What is the estimated blood loss? & $2 / 6(33 \%)$ & $3 / 8(38 \%)$ \\
\hline How long ago was the child born/what was the delivery time? & $2 / 5(40 \%)$ & & $2 / 4(50 \%)$ \\
\hline Clinical information-static & $1 / 5(20 \%)$ & \\
\hline History of high blood pressure? & & \\
\hline Gestational age? & & \\
\hline
\end{tabular}

Abbreviations: IV, intravenous; NICU, neonatal intensive care unit; PPH, postpartum hemorrhage.

Note: Number (percentage) of simulations when the question was repeated during the same scenario. The denominator represents scenarios in which the question was asked at least once.

were to occur, and all participants on the team responded in the role that they occupy in actual practice. Other strengths of the study are intrinsic to the use of simulation. Real-life events often do not lend themselves to an in-depth review of team performance. However, video recorded simulated events allow a repeated review and analysis of all actions and nonactions taken by the team. Simulating clinical events also affords the opportunity to recreate extreme and rarely experienced events for the HCP. By "stressing the system," latent issues that may not be transparent in less stressful situations may become transparent.

It is possible the repeating simulation scenarios could lead to improved communication efficiency and result in decreased repeated questions. In this study, clinicians did not participate in simulated scenarios repeatedly. The use of simulation and debriefing could lead to improved communication for specific scenarios and for general care over time.

This study has several limitations. Simulated clinical scenarios do not reflect actual events with 100\% fidelity. The questions asked in a simulated setting might not be the only pertinent information needed in an actual clinical event. Simulated clinical scenarios may not engender the same degree of physiologic and emotional stress to the level that HCP experience when an actual patient's life is dependent on the care given. Therefore, live clinical events need to be observed to confirm these data. We cannot conclude from this study that repeated questioning would necessarily lead to detrimental patient outcome; we cannot assume that asking for information at various points in clinical care would diminish or improve care. However, improved communication has been established as an important aspect of improving clinical care and patient outcomes. Further, asking the same question unnecessarily is inefficient and potentially precludes a more important question being asked at that same time.

We chose to evaluate three different clinical scenarios. One of our goals was to explore the possibility that there is similar information needed by the team across multiple emergencies. Once identified, that information could then be made readily available during all crises. It is possible that evaluating more iterations of a single simulation could have led to a more robust ability to formulate conclusions based on higher sample size. We encourage other researchers to perform similar studies both in simulation and in actual clinical care to add to our findings.

During an obstetric emergency, communication facilitates both a shared mental model (what are we doing) and role clarity (who is doing it) between the multiple HCPs that typically respond to such events. As described by Weller et al, "Shared mental models lead to a common understanding of the situation, the plan for treatment and the roles and tasks of the individuals in the team." ${ }^{8}$ Without a shared mental model, it is difficult to fully leverage all of the expertise the team can 
provide. However, the use of concise, precise verbal communication during crises is challenging. ${ }^{9}$ This challenge in the health care setting was clearly demonstrated in this study.

Our teams asked a median of 27 questions per 20-minute simulated obstetric emergency-nearly 1.5 questions per minute. Nearly one-third of the questions was repeated at least once; some were repeated multiple times. Many critical obstetric interventions require more than 1 minute to complete; therefore, even the act of asking a relatively simple and brief question could result in misallocation of attention by the HCP during the task saturation that often occurs during a critical event. Further, many time-pressured and high-risk events are characterized by auditory overload-particularly in the small areas that normally comprise the patient care areas on L\&D. Any questions asked by the increasing number of multiple responders-regardless of importance-will contribute to the high decibel level in the delivery room, making it even more difficult to hear the answers. However, certain information is critical and must be shared, especially as responders enter the room and during the acute treatment phase.

This study of simulated obstetric emergencies was able to identify several commonly asked questions across all three emergency events. Defining the information that all team members need to know and then assuring that they have that information in a timely manner will facilitate care of the patient during not only an emergent obstetric event, but any acute event which requires efficient team performance. The development of a variety of modalities including focused team training in communication training and interactive visual displays of key information may ultimately enable HCPs to provide more efficient and effective care leading to optimal patient experiences and outcomes. Future investigations should include prospective observations of live events for lapses in verbal communication, creation of a visual display that is tailored to address the observed lapses, and testing of the display in simulated and ultimately live settings.

\section{Conflict of Interest}

None of the authors has any conflict of interest to disclose.

\section{Acknowledgments}

This project was supported by grant number P30HS023506 from the Agency for Healthcare Research and Quality. The content is solely the responsibility of the authors and does not necessarily represent the official views of the Agency for Healthcare Research and Quality.

\section{References}

1 Pettker CM, Grobman WA. Obstetric safety and quality. Obstet Gynecol 2015;126(01):196-206

2 Preventing infant death and injury during delivery. Jt Comm Perspect 2004;24(09):14-15

3 Lisbon D, Allin D, Cleek C, et al. Improved knowledge, attitudes, and behaviors after implementation of TeamSTEPPS training in an academic emergency department: a pilot report. Am J Med Qual 2016;31(01):86-90

4 Sawyer T, Laubach VA, Hudak J, Yamamura K, Pocrnich A. Improvements in teamwork during neonatal resuscitation after interprofessional TeamSTEPPS training. Neonatal Netw 2013; 32(01):26-33

5 Weld LR, Stringer MT, Ebertowski JS, et al. TeamSTEPPS improves operating room efficiency and patient safety. Am J Med Qual 2016;31(05):408-414

6 Mayer CM, Cluff L, Lin WT, et al. Evaluating efforts to optimize TeamSTEPPS implementation in surgical and pediatric intensive care units. Jt Comm J Qual Patient Saf 2011;37(08):365-374

7 Mann S, Pratt SD. Team approach to care in labor and delivery. Clin Obstet Gynecol 2008;51(04):666-679

8 Weller JM, Torrie J, Boyd M, et al. Improving team information sharing with a structured call-out in anaesthetic emergencies: a randomized controlled trial. $\mathrm{Br} J$ Anaesth 2014;112(06): 1042-1049

9 Brindley PG, Reynolds SF. Improving verbal communication in critical care medicine. J Crit Care 2011;26(02):155-159 